TAIWANESE JOURNAL OF MATHEMATICS

Vol. 14, No. 2, pp. 447-451, April 2010

This paper is available online at http://www.tjm.nsysu.edu.tw/

\title{
FINITENESS RESULT FOR GENERALIZED LOCAL COHOMOLOGY MODULES
}

\author{
Abolfazl Tehranian
}

\begin{abstract}
Let $R$ be a Noetherian ring, let $M$ and $N$ be finitely generated modules and let $\mathfrak{a}$ and $\mathfrak{b}$ be ideals of $R$. Let $s$ be an integer such that $\mathfrak{b}_{\mathfrak{p}} \subseteq$ $\sqrt{\operatorname{Ann} H_{\mathfrak{a}_{\mathfrak{p}}}^{i}\left(M_{\mathfrak{p}}, N_{\mathfrak{p}}\right)}$ for all $i \leq s$ and all prime ideal $\mathfrak{p}$ of $R$. Then we show the following statements hold:

(1) If $\mathrm{H}_{\mathfrak{b}}^{i}(N)=0$ for all $i<s$, then $\mathrm{H}_{\mathfrak{a}}^{i}(M, N)$ is finitely generated for all $i \leq s$.

(2) $\mathfrak{b} \subseteq \sqrt{\operatorname{Ann~} H_{\mathfrak{a}}^{2}(M, N)}$.
\end{abstract}

These statements generalize the corresponding results which are shown in [6] and [1] for standard local cohomology module.

\section{INTRODUCTION}

Throughout this note the ring $R$ is commutative Noetherian with non-zero identity. Let $M$ and $N$ be $R$-modules and let $\mathfrak{a}$ be an ideal of $R$. Then the generalized local cohomology module

$$
\mathrm{H}_{\mathfrak{a}}^{i}(M, N)=\underset{n}{\lim } \operatorname{Ext}_{R}^{i}\left(M / \mathfrak{a}^{n} M, N\right)
$$

was introduced by Herzog in [5] and studied further by Suzuki in [7] and Yassemi in [8]. Note that $\mathrm{H}_{\mathfrak{a}}^{i}(R, N)=\mathrm{H}_{\mathfrak{a}}^{i}(N)$.

Let $M$ be a finitely generated $R$-module and let $\mathfrak{a}, \mathfrak{b}$ be ideals of $R$. In [6] Raghavan proved the following statement:

Received February 12, 2008, accepted June 2, 2008.

Communicated by Wen-Fong Ke.

2000 Mathematics Subject Classification: 13D45, 13 E99.

Key words and phrases: Local cohomology, Modules finite over a local homomorphism, Artinian module, Secondary representation.

Abolfazl Tehranian was supported in part by a grant from Islamic Azad University. 
If $\mathfrak{b}_{\mathfrak{p}} \subseteq \sqrt{\operatorname{Ann} \mathrm{H}_{\mathfrak{a}_{\mathfrak{p}}}^{1}\left(M_{\mathfrak{p}}\right)}$ for every prime ideal $\mathfrak{p}$ of $R$, and $\mathfrak{b} \subseteq \sqrt{\operatorname{AnnH}_{\mathfrak{a}}^{0}(M)}$, then $\mathfrak{b} \subseteq \sqrt{\operatorname{Ann} \mathrm{H}_{\mathfrak{a}}^{1}(M)}$. If, in addition, $\mathrm{H}_{\mathfrak{b}}^{0}(M)=\mathrm{H}_{\mathfrak{a}}^{0}(M)=0$, then $\mathrm{H}_{\mathfrak{a}}^{1}(M)$ is finitely generated.

On the other hand in [1] Brodmann, Rotthaus, and Sharp proved the following statement:

If $\mathfrak{b}_{\mathfrak{p}} \subseteq \sqrt{\operatorname{Ann~}_{\mathfrak{a}_{\mathfrak{p}}}^{i}\left(M_{\mathfrak{p}}\right)}$ for all $i \leq 2$ and all prime ideal $\mathfrak{p}$ of $R$ then $\mathfrak{b} \subseteq$ $\sqrt{\operatorname{Ann~}_{\mathfrak{a}}^{i}(M)}$ for all $i \leq 2$.

In this note we generalize the above results for the generalized local cohomology modules, see Theorems 1 and 2.

\section{MAIN RESULTS}

First we recall some known results which we will use in this paper.

Lemma A. ([1, Lemma 2.1]). Let $L$ be an $R$-module such that the set of

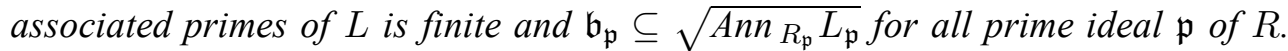
Then $\mathfrak{b} \subseteq \sqrt{\operatorname{Ann}_{R} L}$.

Theorem B. ([9, Theorem 2.1]). Let $M$ and $N$ be finitely generated $R$ modules. Let $s \in \mathbb{N}_{0}$ be such that $H_{\mathfrak{a}}^{i}(M, N)$ is finitely generated for all $i<s$. Then the set of associated primes of the module $H_{\mathfrak{a}}^{s}(M, N)$ is finite.

In the first step we prove an extension of Faltings' lemma cf. [3, Lemma 3] and [4, pp. 48-49], (see also [2, Prop. 9.1.2] and [2, Theorem 9.6.1]), in the context of generalized local cohomology modules.

Theorem 1. Let $M$ and $N$ be finitely generated $R$-modules and let $s \in \mathbb{N}$. Then the following statements are equivalent:

(1) $H_{\mathfrak{a}}^{i}(M, N)$ is finitely generated for all $i<s$;

(2) $H_{\mathfrak{a}_{\mathfrak{p}}}^{i}\left(M_{\mathfrak{p}}, N_{\mathfrak{p}}\right)$ is finitely generated for all $i<s$ and all prime ideal $\mathfrak{p}$ of $R$;

(3) $\mathfrak{a} \subseteq \sqrt{\operatorname{Ann} H_{\mathfrak{a}}^{i}(M, N)}$ for all $i<s$.

Proof. We show that $(1) \Leftrightarrow(2)$ and $(1) \Leftrightarrow(3)$.

$(1) \Rightarrow(2)$ is clear.

$(1) \Rightarrow(3)$ : It follows from the fact that $\mathrm{H}_{\mathfrak{a}}^{i}(M, N)$ is finitely generated and $\mathfrak{a}-$ torsion.

(3) $\Rightarrow(1)$ : We use induction on $s$. For $s=1$ the assertion follows from the fact $\mathrm{H}_{\mathfrak{a}}^{0}(M, N)=\Gamma_{\mathfrak{a}}(\operatorname{Hom}(M, N)$. Let $s>1$. By induction hypothesis we know that 
$\mathrm{H}_{\mathfrak{a}}^{i}(M, N)$ is finitely generated for $0 \leq i \leq s-2$. Hence it remains to prove that $\mathrm{H}_{\mathfrak{a}}^{s-1}(M, N)$ is finitely generated. First assume that $N$ is $\mathfrak{a}$-torsion free module. Let $x$ be an element of $\mathfrak{a}$ which is regular on $N$. Then $x^{k} \mathrm{H}_{\mathfrak{a}}^{s-1}(M, N)=0$ for all $k \gg 0$. It follows from [2, Lemma 9.1.1] and the long exact sequence on generalized local cohomology induced by $0 \longrightarrow N \stackrel{x^{k} .}{\longrightarrow} N \longrightarrow N / x^{k} N \longrightarrow 0$ that $\mathfrak{a} \subseteq \sqrt{\operatorname{Ann}_{\mathfrak{a}}^{i}\left(M, N / x^{k} N\right)}$ for all $i<s-1$. Hence by induction hypothesis $\mathrm{H}_{\mathfrak{a}}^{i}\left(M, N / x^{k} N\right)$ is finitely generated for all $i<s-1$. Thus $\mathrm{H}_{\mathfrak{a}}^{s-1}(M, N)$ is finitely generated (since it is homomorphic image of $\mathrm{H}_{\mathfrak{a}}^{s-2}\left(M, N / x^{k} N\right)$ ).

Now let $N$ be an arbitrary finitely generated $R$-module, and set $L=\Gamma_{\mathfrak{a}}(N)$, $K=N / L$. The exact sequence $0 \rightarrow L \rightarrow N \rightarrow K \rightarrow 0$ induces the exact sequence $\mathrm{H}_{\mathfrak{a}}^{i}(M, L) \rightarrow \mathrm{H}_{\mathfrak{a}}^{i}(M, N) \rightarrow \mathrm{H}_{\mathfrak{a}}^{i}(M, K) \rightarrow \mathrm{H}_{\mathfrak{a}}^{i+1}(M, L)$. By [9, Lemma 1.1] we know that $\mathrm{H}_{\mathfrak{a}}^{i}(M, L)=\operatorname{Ext}^{i}(M, L)$ for all $i$ and so $\mathfrak{a} \subseteq \sqrt{{\operatorname{AnnH_{\mathfrak {a}}}}^{i+1}(M, L)}$. Thus $\mathfrak{a} \subseteq \sqrt{\left.\operatorname{Ann}_{\mathfrak{a}}^{i}(M, K)\right)}$ for all $i<s$. Now since $K$ is $\mathfrak{a}$-torsion free we have that $\mathrm{H}_{\mathfrak{a}}^{i}(M, K)$ is finitely generated for $i<s$ and hence $\mathrm{H}_{\mathfrak{a}}^{i}(M, N)$ is finitely generated for $i<s$.

(2) $\Rightarrow(1)$ : For $s=1$ it is clear. Let $s>1$. By the induction hypothesis we have $\mathrm{H}_{\mathfrak{a}}^{i}(M, N)$ is finitely generated for $0 \leq i \leq s-2$ and hence $\left|\operatorname{Ass~}_{\mathfrak{a}}^{i}(M, N)\right|<\infty$ for all $i \leq s-1$ by Theorem B. By Lemma A we have $\mathfrak{a} \subseteq \sqrt{\operatorname{Ann}_{\mathfrak{a}}^{i}(M, N)}$ for all $i<s-1$. Now the assertion follows from the equivalence between (1) and (3).

The following theorem is a generalization of the main result in [6].

Theorem 2. Let $M$ and $N$ be finitely generated modules and let $\mathfrak{a}$ and $\mathfrak{b}$ be ideals of $R$. If $s$ is an integer such that $H_{\mathfrak{b}}^{i}(N)=0$ for $i<s$, and $\mathfrak{b}_{\mathfrak{p}} \subseteq$ $\sqrt{\text { Ann } H_{\mathfrak{a}_{\mathfrak{p}}}^{i}\left(M_{\mathfrak{p}}, N_{\mathfrak{p}}\right)}$ for all $i \leq s$ and all prime ideal $\mathfrak{p}$ of $R$ then $H_{\mathfrak{a}}^{i}(M, N)$ is finitely generated for all $i \leq s$.

Proof. We use induction on $s$. Assume that $s=1$. Since $\mathrm{H}_{\mathfrak{p}}^{0}(M, N)=$ $\Gamma_{\mathfrak{a}}\left(\operatorname{Hom}(M, N)\right.$ is finitely generated, the set of associated primes of $\mathrm{H}_{\mathfrak{a}}^{1}(M, N)$ is finite by Theorem $\mathrm{B}$, and hence by Lemma $\mathrm{A}$ we have that $\mathfrak{b} \subseteq \sqrt{\operatorname{Ann~}_{\mathfrak{a}}^{1}(M, N)}$. Therefore, there exists an integer $k$ such that $\mathfrak{b}^{k} \mathrm{H}_{\mathfrak{a}}^{1}(M, N)=0$. Let $x$ be an element of $\mathfrak{b}$ that is regular on $N$. Since $x^{k} \mathrm{H}_{\mathfrak{a}}^{1}(M, N)=0$, it follows from the long exact sequence on generalized local cohomology induced by $0 \longrightarrow N \stackrel{x^{k}}{\longrightarrow}$ $N \longrightarrow N / x^{k} N \longrightarrow 0$ that $\mathrm{H}_{\mathfrak{a}}^{1}(M, N)$ is a homomorphic image of $\mathrm{H}_{\mathfrak{a}}^{0}\left(M, N / x^{k} N\right)$ and hence is finitely generated.

Now let $s>1$. By induction hypothesis we have $\mathrm{H}_{\mathfrak{a}}^{i}(M, N)$ is finitely generated for all $i<s$ and hence $\operatorname{Ass~}_{\mathfrak{a}}^{s}(M, N)$ is a finite set by Theorem B. Let $x$ be an element in $\mathfrak{b}$ that is regular on $N$. By Lemma A we know that $x^{k} \mathrm{H}_{\mathfrak{a}}^{s}(M, N)=0$ for all $k \gg 0$. It follows from the long exact sequence on local cohomology induced by $0 \longrightarrow N \stackrel{x^{k}}{\longrightarrow} N \longrightarrow N / x^{k} N \longrightarrow 0$ that $H_{\mathfrak{b}}^{i}\left(N / x^{k} N\right)=0$ for all $i<s-1$. Now 
it follows from the long exact sequence

$$
\mathrm{H}_{\mathfrak{a}}^{i}(M, N) \longrightarrow \mathrm{H}_{\mathfrak{a}}^{i}\left(M, N / x^{k} N\right) \longrightarrow \mathrm{H}_{\mathfrak{a}}^{i+1}(M, N) \stackrel{x^{k}}{\longrightarrow} \mathrm{H}_{\mathfrak{a}}^{i+1}(M, N)
$$

and using [2, Lemma 9.1.1], we have $\mathfrak{b}_{\mathfrak{p}} \subseteq \sqrt{\operatorname{Ann}_{\mathfrak{a}_{\mathfrak{p}}}^{i}\left(M_{\mathfrak{p}},\left(N / x^{k} N\right)_{\mathfrak{p}}\right)}$ for all $i<s$. Therefore, by induction hypothesis $\mathrm{H}_{\mathfrak{a}}^{i}\left(M, N / x^{k} N\right)$ is finitely generated for all $i<s$ and hence $\mathrm{H}_{\mathfrak{a}}^{s}(M, N)$ is finitely generated ( since it is a homomorphic image of $\left.\mathrm{H}_{\mathfrak{a}}^{s-1}\left(M, N / x^{k} N\right)\right)$.

Corollary 3. Let $M$ and $N$ be finitely generated modules and let $\mathfrak{a}$ and $\mathfrak{b}$ be ideals of $R$. If $\mathfrak{b}_{\mathfrak{p}} \subseteq \sqrt{\text { Ann } H_{\mathfrak{a}_{\mathfrak{p}}}^{i}\left(M_{\mathfrak{p}}, N_{\mathfrak{p}}\right)}$ for all $i \leq 2$ and all prime ideal $\mathfrak{p}$ of $R$ then $\mathfrak{b} \subseteq \sqrt{\operatorname{Ann} H_{\mathfrak{a}}^{i}(M, N)}$ for all $i \leq 2$.

Proof. Set $N^{\prime}=N / \Gamma_{\mathfrak{b}}(N)$. By using the same technique as [1, Remark 1.3(ii)], we have that $\mathfrak{b}_{\mathfrak{p}} \subseteq \sqrt{\operatorname{Ann}_{\mathfrak{a}_{\mathfrak{p}}}^{i}\left(M_{\mathfrak{p}}, N_{\mathfrak{p}}^{\prime}\right)}$ for all $i \leq 2$ and all prime ideal $\mathfrak{p}$ of $R$. Since $\mathrm{H}_{\mathfrak{b}}^{i}\left(N^{\prime}\right)=0$ for all $i<1$ we have $\mathrm{H}_{\mathfrak{a}}^{i}\left(M, N^{\prime}\right)$ is finitely generated for all $i \leq 1$ and hence $\operatorname{Ass~}_{\mathfrak{a}}^{2}\left(M, N^{\prime}\right)$ is a finite set. Therefore, by Lemma A, $\mathfrak{b} \subseteq \sqrt{\operatorname{Ann}_{\mathfrak{a}}^{i}(M, N)}$ for all $i \leq 2$.

\section{ACKNOWLEDGMENT}

The author would like to thank the referee for his/her useful comments.

\section{REFERENCES}

1. M. Brodmann, Ch. Rotthaus and R. Y. Sharp, On annihilators and associated primes of local cohomology modules, J. Pure Applied Algebra, 153 (2000), 197-227

2. M. Brodmann and R. Sharp, Local cohomology-an algebraic introduction with geometric applications, Cambridge studies in advanced Mathematics No. 60, Cambridge University Press, 1998.

3. G. Faltings, Über die Annulatoren lokaler Kohomologiegruppen, Arch. Math., 30 (1978), 473-476.

4. G. Faltings, Der Endlichkeitssatz der lokalen kohomologie, Math. Ann., 255 (1981), 45-56.

5. J. Herzog, Komplexe, Auflösungen und dualität in der lokalen Algebra, Preprint 1974, Univ. Regensburg.

6. K. N. Raghavan, Local-global principle for annihilation of local cohomology, Commutative algebra: syzygies, multiplicities, and birational algebra (South Hadley, MA, 1992), 329-331, Contemp. Math., 159, Amer. Math. Soc., Providence, RI, 1994. 
7. N. Suzuki, On the generalized local cohomology and its duality, J. Math. Kyoto Univ., 18 (1978), 71-85.

8. S. Yassemi, Generalized section functor, J. Pure Applied Algebra, 95 (1994), 103119.

9. S. Yassemi, L. Khatami and T. Sharif, Associated primes of generalized local cohomology modules, Comm. Algebra, 30 (2002), 327-330.

\author{
Abolfazl Tehranian \\ Science and Research Branch, \\ Islamic Azad University, \\ Tehran, Iran \\ E-mail: tehranian1340@yahoo.com
}

